behaviour of the cleaners. Byusing the pulleys, Bshary and Grutter could test whether cleaner fish were more likely to take the less preferred food, if taking the more preferred food from a plate meant that they would lose access to other plates (just as they would lose access to other clients in the wild if they cheated). And indeed, the authors did find audience effects under these admittedly contrived conditions, as cleaners were induced into eating the less preferred foods.

If social networks such as those uncovered by Bshary and Grutter in fish are common in other animals, the prospect for researchers is both exciting and daunting. It is exciting because it suggests that animal behaviour has to be seen as being embedded in a rich social tapestry, composed of interconnected direct and indirect streams of information. And it is logistically daunting for exactly the same reason. Lee Alan Dugatkin is in the Department of Biology, University of Louisville, Louisville, Kentucky 40202, USA.

e-mail:lee.dugatkin@louisville.edu

1. Bshary, R. \& Grutter. A. S Nature 441,975-978 (2006)

2. Alexander R. The Bilagyy of Moral Systems(Aldine De Gruyter, New York, 1987)

3. Nowek, M.A. \& Sigmund, K Nature 393,573-577 (1998).

4. Wedekind, C\& Milinski, M. Science 288, 850-852 (2000).

5. Pollock, G \&Dugatkin, L.A.J. Thear. Bial. 159,25-37 (1992)

6. Zahavi, A.\& Zahavi, A. The Handicap Pinciple (Ox fordUniv. Press, 1997).

7. McGregor, P. (ed) Animal Communication Networks (Cambridge Univ. Press, 2005).

\title{
ASTROPHYSICS
}

\section{Magnetic accretion}

\section{Daniel Proga}

\section{Disks of hot gas drawn onto a central star or black hole are the best energy- producing machines in the Universe. So how do these accretion disks work? The answer, it seems, is blowing in their winds.}

Astronomical objects that siphon off their energy from gravitational processes are the most powerful sources of electromagnetic radiation in the Universe. Of these, quasi-stellar radio sources (quasars), which can release as much power as several hundred galaxies, are perhaps the most spectacular example. But for all their brightness, gravity-powered sources are secretive when it comes to revealing how exactly they produce their radiation. On page 953 of this issue, Miller et al. ${ }^{1}$ investigate a system known as GRO J1655-40, in which a black hole of seven solar masses accretes gas stripped from a normal star. They observe not just radiation, but a wind of ionized gas emerging from the system, and conclude that a magnetic process powers this wind - and that this process could also play the lead part in producing the radiation.

For astronomers, gravity is not just the seemingly all-conquering force of everyday experience, but also the ultimate source of energy. In a star depleted of nuclear fuel, for instance, gravity prevails to form a very dense, compact object. Depending on the star's initial mass, this object could be a white dwarf, a neutron star or a black hole. When rotating gas is fed to such a compact object, as for example in GRO J1655-40, its mass is converted to radiation with an efficiency of a few per cent. That climbs to $40 \%$ for a rapidly rotating black hole - values significantly higher than the $1 \%$ conversion efficiency for nuclear fusion ${ }^{2}$.

So how do these cosmic engines work? Initially, the falling gas forms a thin, dense accretion disk' that encircles the compact object and is supported against gravitational collapse by centrifugal force. Through viscous damping processes within the disk, the mechanical energy of the gas is dissipated and radiated away as continuum emission over all wavelengths. That much is understood; what is unclear, however, is how exactly the mechanical energy is converted into heat and radiation energy. Moreover, for gas to accrete throughout the centrifugally supported disk, conservation of angular momentum requires a mechanism that transfers the gas's angular momentum rapidly outwards. Otherwise, the gas would orbit the central object forever, and no radiation would be produced.

For decades, hydrodynamic processes were thought to supply this mechanism. But despite some successes - such as obtaining an understanding of the role of turbulence in disk dynamics - the hydrodynamical approach has so far not produced a consistent model that can be derived from first principles ${ }^{3,4}$. Only a turbulent disk with interacting eddies will supply an effective viscosity large enough to drive the fast accretion rates observed in disks, but the question of what powers the turbulence remains unanswered.

In this context, the introduction of magnetic fields, presumed to originate in the star feeding the compact object, pays off handsomely. This applies even to small fields that are hard to measure directly: through an effect known as magneto-rotational instability ${ }^{5}$, a weak magnetic field can act as a kind of spring connecting inner and outer areas of gas in the disk. This spring exerts a force that transfers angular momentum and energy outwards and extracts disk rotational energy to power turbulence.

Miller and colleagues ${ }^{1}$ find indirect evidence to support this theory in the properties of a wind driven from the accretion disk of GRO J1655-40. They studied an X-ray spectrum of the system taken by NASA's Chandra satellite observatory and detected tens of spectral absorption lines shifted towards shorter wavelengths. Such a Glueshift' indicates that absorbing gas is moving towards the observer and, using geometrical and physical arguments, Miller et al. conclude that the absorbing medium is a wind blowing outwards from the accretion disk at a small angle to the disk's plane. The fact that the wind is blowing from the disk and not, for example, from the companion star means that the wind's properties can reveal the properties of the disk itself.

To produce such a wind, the upper disk atmosphere must gain extra energy to overcome gravity. This extra energy can come from heating, from radiation pressure, from magnetic fields or from any combination of the three $^{3,67}$. In many systems, it is hard to distinguish which of these dominates. Happily, Miller et al. argue ${ }^{1}$, the GRO J1655-40 case is clear-cut. In this system, the wind is too cold for its thermal pressure to overcome gravity, and the radiation pressure is too weak to drive a wind. Thus, by a process of elimination, a magnetic origin for the wind is the only option. This is an attractive possibility, as theories exist that posit magnetic fields as the drivers of disk accretion in the first place ${ }^{7 s}$, and a larger mystery would therefore also be solved.

Not all might find Miller and colleagues' arguments for magnetic fields as the drivers of disk accretion convincing, partly because they rely on evidence that is mostlyindirect. But this is the best that can be done at the moment: current models lack the predictive power to demonstrate that magnetic fields power the wind and disk accretion that are responsible, respectively, for the observed absorption lines and continuum emission. Nevertheless, many astronomers will be motivated by such work to develop and explore this promising model. Clearly, much can be learned about accretion disks by studying the properties of what escapes them - notonly radiation, but gas, too. Daniel Proga is in the Physics Department, University of Nevada Las Vegas, 4505 South Maryland Parkway, Las Vegas,

Nevada 89154-4002, USA. e-mail:dproga@physics.unlv.edu

1. Miller, 1 M. et al. Nature 441, 953-955(2006)

2. Frank, L, King A. \& Raine, D. Accretion Power in Astrophysics (Cambridge Univ. Press, 2002)

3 Shakura, N. L \& Sunyaev, R.A. Astron Astrophys. 24, 337-355 (1973).

4. Balbus, S. A. \& Hawley, J.F.Rex Mod Phys. 70, 1-53(1998)

5. Balbus, S A. \& Hawley, J.F.Astrophys J.376, 214-233 (1991).

6. Begelman M. C etal. Astrophys J. 277, 70-89 (1983).

7. Blandford R. D.\& Payne, D. G. Mon Not. R.Astron.Soc. 199, 883-903 (1982).

8. Miller, K.A. \& Stone, J.M. Astraphys J. 534,398-419 (2000) 